\title{
Enriching for HIV-infected cells using anti-gp41 antibodies indirectly conjugated to magnetic microbeads
}

\author{
Bouchaib Bahbouhi and Lena Al-Harthi
}

BioTechniques 36:139-147( January 2004)

\begin{abstract}
The isolation of a pure population of human immunodeficiency virus (HIV)-infected cells is highly desirable for evaluating the impact of HIV on cellular gene expression. Given that HIV gp41 transmembrane protein is anchored on the surface of HIV-infected cells, we evaluated the use of pooled anti-gp41 monoclonal antibodies (MAbs) and HIV immunoglobulins (HIV-Igs) indirectly conjugated to magnetic microbeads to positively select for infected cells. We demonstrate that pooled anti-gp41 monoclonal antibodies enriched for $\mathrm{H} 9$ cells infected with HIV IIIB by approximately 98\%. Peripheral blood mononuclear cells (PBMCs) infected with a primary (HIV strain 302151) or laboratory-adapted (IIIB) strain were enriched by 54\%-62\%, depending on the initial viral inoculum. Using HIV-Ig in this magnetic positive-selection approach was also highly efficient for enriching for H9 cells infected with $I I I B$ but less efficient for infected PBMCs. Both types of antibodies used in the selection process resulted in $>80$ viability of selected $H I V$-infected cells. Analysis of interleukin 2 (IL-2) mRNA expression using real-time reverse transcription PCR (RT-PCR) of the $H I V$-enriched population demonstrated a higher level of IL-2 mRNA, by approximately four cycles, and an 8-fold increase in IL-2 expression, as evaluated by intracellular staining and flow cytometric analysis, in comparison to gp41-negative cells. Collectively, these data illustrate that antibodies targeting gp41 can be used to enrich for HIV-positive populations. This represents a novel approach for studying the impact of HIV on infected cells and on bystander/uninfected cells.
\end{abstract}

\section{INTRODUCTION}

Several mechanisms, including direct and indirect mechanisms, exist by which human immunodeficiency virus (HIV) is known to mediate its pathogenecity, including altering host cell properties $(1,2)$. Studies that have evaluated this impact have relied on mixed populations of infected and uninfected cells (3-8). Classically, the cells are infected with HIV or mock-infected, and these two populations are compared. However, not every cell, even susceptible T cells, exposed to HIV becomes infected. In this scenario, the net effect may not be due to infected cells but due to a bystander effect $(9,10)$. It is, therefore, critical to devise strategies to be able to specifically pull out only the infected population.
To date, a number of reports have discriminated between infected and uninfected cells by one of two approaches: utilizing HIV-1 tagged with a reporter protein [most commonly used is the green fluorescent protein (GFP) $(11,12)$ or the mouse heat shock protein (HAS) $(13,14)]$ or intracellular detection of HIV-1 core p24 antigen $(13,15)$. These approaches have several disadvantages. The detection of p24 requires the permeabilization of the cells to detect this intracellular protein, which makes its use prohibitive to the isolation of viable cells for further in vitro culturing. GFP does not require permeabilization of cells to detect its signal but, as in the case of HAStagged viruses, its use requires the cloning of the specific HIV strain into these reporter constructs, thus limiting the analyses to those available HIVtagged strains or requiring the cloning of the particular isolate of interest first into these reporter constructs. Cloning can be cumbersome to perform for multiple primary HIV isolates and often requires the deletion of some region of the viral gene (e.g., nef or vpr) to insert the reporter gene. Live cell sorting using a flow cytometer can be used to isolate GFP-positive or HAS-positive HIV-infected cells. The disadvantage of this approach is the high cost of a flow cytometer with live cell sorting capabilities and the need to perform these studies in a biologic level three biohazard containment facility to protect against aerosol. GFP-positive cells cannot be pulled out using less expensive magnetic bead isolation protocols because the protein is intracellular. 
We utilized anti-gp41 antibodies indirectly conjugated to magnetic microbeads to enrich for HIV-infected populations for the following reasons. (i) gp41 is a highly hydrophobic protein that is not shed in the culture medium of both primary and $\mathrm{T}$ cell line-adapted viruses and remains stably expressed on the cell surface of infected cells during multiple rounds of viral replication (16); (ii) HIV gp41 can be detected intracellularly as it is being assembled within the cell post-productive infection (17); and (iii) numerous anti-gp41 monoclonal antibodies have been characterized, some of which are directly isolated from infected patients (18). Here we describe the enrichment of peripheral blood mononuclear cells (PBMCs) and a $\mathrm{T}$ cell line (H9) infected with either primary or T-tropic isolates of HIV. We then used the gp41-selected cell fraction to evaluate the impact of direct HIV infection on interleukin 2 (IL-2) transcript level using real-time reverse transcription PCR (RT-PCR).

\section{MATERIALS AND METHODS}

\section{Materials}

Uninfected H9 cells and H9 cells chronically infected with HIV-1 IIIB (H9-IIIB) were obtained from the AIDS Research and Reference Reagent Program (Division of AIDS, NIAID, National Institutes of Health, Bethesda, MD, USA). The cells were propagated in RPMI medium supplemented with $10 \%$ heat-inactivated fetal bovine serum (FBS), $100 \mathrm{U} / \mathrm{mL}$ penicillin, $100 \mu \mathrm{g} / \mathrm{mL}$ streptomycin, and $2 \mathrm{mM} \mathrm{L}$-glutamine. PBMCs from healthy blood donors were isolated by density gradient centrifugation from heparinized blood within $1 \mathrm{~h}$ of venipuncture. PBMCs were cultured as described for the $\mathrm{H} 9$ cells, but with the addition of IL-2 at $20 \mathrm{U} / \mathrm{mL}$.

H9 or PBMCs were infected with HIV IIIB or primary HIV strain 302151 (AIDS Research and Reference Reagent Program), respectively, at either high viral inoculum (20 ng HIV p24/1 $\times 10^{6}$ cells overnight) or lower viral inoculum (5 ng HIV p24/ $1 \times 10^{6}$ cells for $4 \mathrm{~h}$ ) at $37^{\circ} \mathrm{C}$. Subsequently, HIV-exposed cultures or mock-infected cultures were washed twice to remove unbound virus and cultured as described above. HIV-infected PBMCs were stimulated with 2 $\mu \mathrm{g} / \mathrm{mL}$ phytohemagglutinin (PHA) and cultured in the presence of $20 \mathrm{U} / \mathrm{mL}$ IL-2. The medium was changed every 3 days with IL-2 replenishment. On the 6th day post-infection, the cells were stimulated again with PHA to maintain viral replication by augmenting cell activation. HIV IIIB-infected cells were examined daily for viral mediated-syncytia induction, and the medium was changed every 3 days. The collected supernatants were centrifuged at $300 \times$ $g$ to remove cell debris and measured for p24 antigen production using standard ELISA (AIDS Vaccine Program, Frederick, MD, USA). HIV infection was also detected by intracellular staining of p24 from an aliquot of infected cells using flow cytometry.

Monoclonal antibodies (MAbs) CD4 and CD3 conjugated to phycoerythrin (PE), fluorescein isothiocyanate (FITC), peridinin chlorophyll protein (PerCP), or allophycocyanin (APC) and mouse anti-human immunoglobulin $\mathrm{G}$ ( $\mathrm{IgG})$ coupled to $\mathrm{PE}$ or $\mathrm{Cy}-\mathrm{Chrome}^{\mathrm{TM}}$ were purchased from BD Pharmingen (San Diego, CA, USA). Anti-HIV-1 core p24 antigen conjugated to FITC or PE was purchased from Beckman Coulter (Fullerton, CA, USA). Human antiHIV-1 gp41 antibodies (2F5, 5F3, F240, 50-69, and 240-D) and HIV immunoglobulin (HIV-Ig) were obtained from the AIDS Research and Reference Reagent Program. The 2F5 and 5F3 were provided by Dr. Herman Katinger; F240 was provided by Drs. Marshall Posner and Lisa Cavacini; 50-69 and 240-D were provided by Dr. Susan ZollaPazner; and HIV-Ig was provided from human plasma of infected patients from Nabi Biopharmaceuticals (Boca Raton, FL, USA). The 5F3 antibody reacts with amino acids 526-543 of the gp41 protein, while the remaining antibodies recognize overlapping epitopes between amino acids 579-613.

\section{Immunostaining and Flow Cytometric Analysis of Cell Surface and Intracellular Proteins}

Intracellular staining of HIV-p24 was performed using the FIX \& PERM ${ }^{\circledR}$ kit (Caltag Laboratories, Burlingame, CA,
USA), according to the protocol provided by the manufacturer of HIV-p24 (BD Biosciences, San Jose, CA, USA). For the detection of cell-associated HIV-1 gp41 (cell surface-bound and intracellular gp41), we performed an indirect intracellular immunostaining procedure. Briefly, $F c$ receptors were blocked by incubating the cells with 1 $\mathrm{mL}$ of inactivated human serum from HIV-negative donors for $30 \mathrm{~min}$ at room temperature with gentle shaking or, alternatively, with $20 \mu \mathrm{L} F c$ receptor blocking reagent (BD Biosciences). The cells were then washed, fixed, and permeabilized in the presence of $5 x$ pooled anti-gp41 MAbs in $100 \mu \mathrm{L}$ permeabilization buffer (Caltag Laboratories). The amount of antibodies $(10 \mu \mathrm{L}$ each for a total of $50 \mu \mathrm{L}$ ) in the final $1 \times$ pooled mixture represents 4.6, 0.8, 0.4, and $15 \mu \mathrm{g}$ for $2 \mathrm{~F} 5, \mathrm{~F} 240,240 \mathrm{D}$, and $5 F 3$, respectively. The 50-69 monoclonal is a supernatant provided with no designation of concentration, and it was also used at $10 \mu \mathrm{L}$ in a total of 50 $\mu \mathrm{L}$, representing a 5 -fold dilution of the original 50-69 stock in the pooled MAb mixture. After washing, $5 \mu \mathrm{L}$ mouse anti-human IgG-PE (or Cy-Chrome) and $20 \mu \mathrm{L}$ anti-CD4-FITC (or APC) antibodies were added to the cells in permeabilization buffer and then incubated at room temperature for $20 \mathrm{~min}$. The cells were subsequently washed with PBS and fixed with 2\% formaldehyde. Detection of surface-bound gp41 was performed by blocking $F c$ receptors and then incubating the cells with pooled gp41 MAbs diluted to $1 / 10$ volume in the binding buffer [phosphate-buffered saline (PBS), $0.5 \%$ bovine serum albumin (BSA), and 2 mM EDTA, pH 7.2] at room temperature for $30 \mathrm{~min}$. After washing, the cells were incubated with anti-human PE (Cy-Chrome) and antiCD4 as described.

Intracellular IL-2 protein expression was evaluated by flow cytometry. Specifically, the initial and gp41-selected cell fractions were incubated with Brefeldin A (BFA) solution (BD Pharmingen) for 3-4 $\mathrm{h}$ to block cytokine production. Conventional permeabilization and intracellular staining using IL-2-APC and p24-PE were subsequently performed according to the manufacturer's instructions (BD Biosciences). 


\section{Enriching for HIV-1-Infected Cells}

Eight days post-infection, at least $10 \times 10^{6}$ infected cells (pooled from separate wells each containing $2 \times 10^{6}$ infected cells) or mock-infected cells were collected by centrifugation and washed in binding buffer. $F c$ receptors were blocked as described above. The cells were then washed and incubated with $5 \times$ pooled anti-gp41 human MAbs (final volume was $10 \mu \mathrm{L}$ of the pooled mixture) or HIV-Ig $(50 \mu \mathrm{g})$ in $200 \mu \mathrm{L}$ binding buffer for 45-60 min at room temperature with shaking. The cells were then washed with cold binding buf- fer by centrifugation at $4^{\circ} \mathrm{C}$, resuspended with $20 \mu \mathrm{L}$ mouse anti-human IgG microbeads (Miltenyi Biotec $\mathrm{GmbH}$, Bergisch, Gladbach, Germany) in 200 $\mu \mathrm{L}$ binding buffer, and incubated at $4^{\circ} \mathrm{C}$ for 20 min with shaking. The cells were subsequently washed in cold PBS, and one aliquot was removed from the infected cells for further analysis (initial fraction). Both infected and mock-infected cells were subjected to magnetic bead separation using the autoMACS ${ }^{\mathrm{TM}}$ cell separation apparatus (Miltenyi Biotec), specifically using the double-positive selection (Posseld) program.

The saved aliquot of the starting fraction and the isolated negative and positive fractions of both infected and mock-infected cells were analyzed for cell viability using the trypan blue exclusion assay and analyzed for enrichment for infected cells by immunostaining for gp41 and/or p24, as described earlier. In all flow cytometry analyses, CD3 and CD4 (FITC, PE, PerCP, and APC) were used to compensate for overlapping signals. Quadrants were set using mock-infected cells that were treated under the same experimental conditions. Data analysis was performed using CellQuest ${ }^{\mathrm{TM}}$ (BD Biosciences) software.

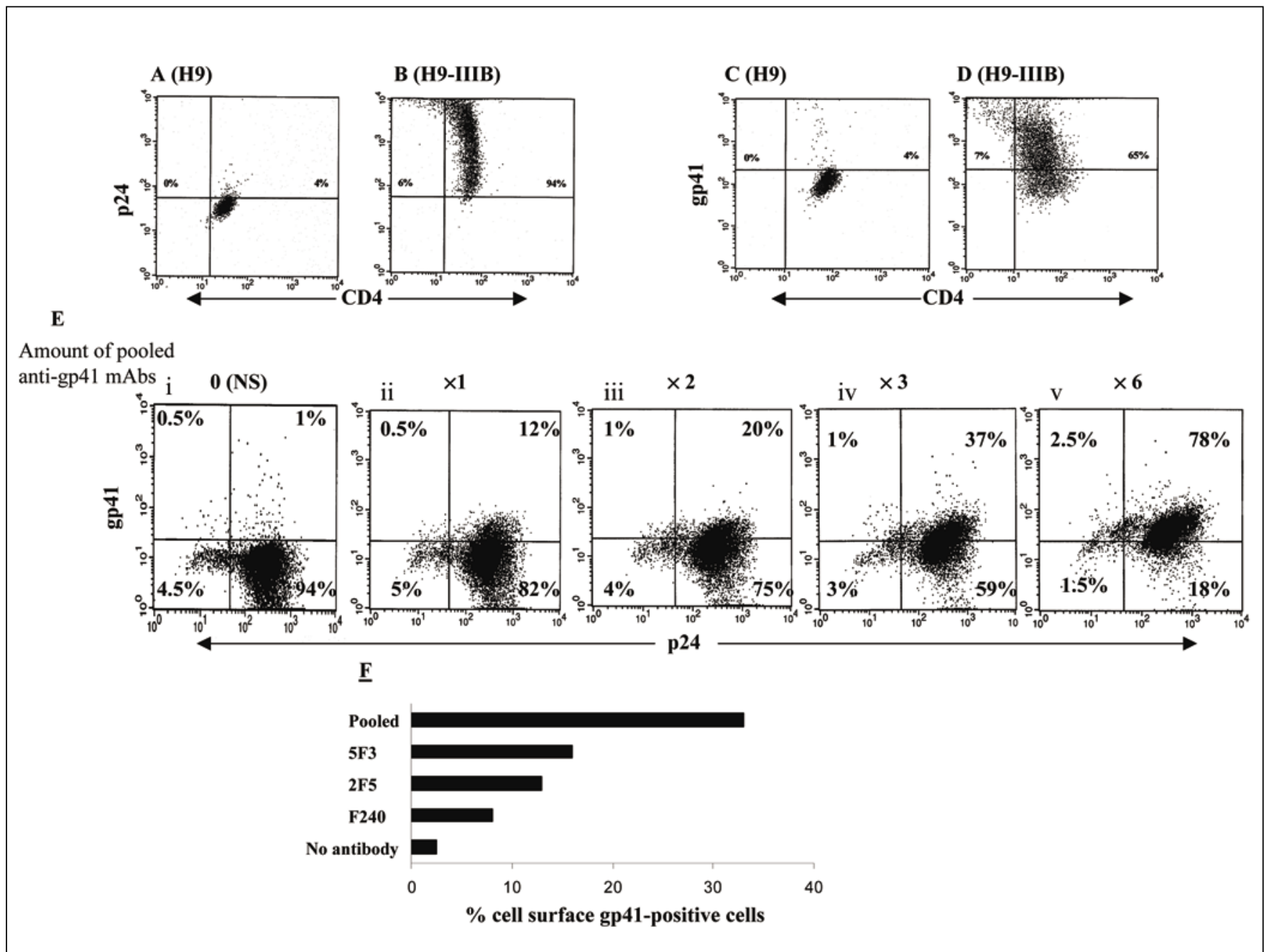

Figure 1. Intracellular detection of p24 and gp41 by flow cytometry. H9-IIIB cells chronically infected with human immunodeficiency virus type 1 (HIV-1) and their parental uninfected H9 cells were stained for anti-CD4-fluorescein isothiocyanate (FITC) and either (A and B) anti-HIV p24-phycoerythrin (PE) or (C and D) $5 \times$ pooled anti-gp41 monoclonal antibodies (MAbs) indirectly conjugated to anti-human PE. The amount of antibodies in the $1 \times$ anti-gp41 pooled mixture is described in the text. Pooled antibodies were used to stain H9-IIIB cells at $1 \times($ E-ii), $2 \times$ (E-iii), $3 \times$ (E-iv), and $6 \times(v)$. Nonspecific (NS) binding to gp41 was evaluated by incubating the cells with human serum that was negative for HIV (E-i). Each anti-gp41 MAb was used to stain H9-IIIB cells at the same concentration as in the $5 \times$ MAbs pool. This antibody amount was selected on the basis of the minimum amount necessary to detect gp41 (higher amounts could not be used because of the limited quantities supplied of each antibody). (F) The efficiency of the pooled anti-gp41 MAbs was compared to single MAbs for detecting cell surface gp41. All samples were analyzed by flow cytometry using uninfected cells to set the quadrants. Data are representative of at least three experiments. 


\section{Detection of IL-2 in gp41-Selected Cells by Real-Time RT-PCR}

Total RNA was extracted from mockinfected and magnetically selected HIV1-positive cells (gp41-positive fraction) using the NucleoSpin ${ }^{\circledR}$ RNA purification kit (BD Biosciences Clontech, Palo Alto, CA, USA). Total RNA was then used for mRNA purification using NucleoTrap ${ }^{\circledR}$ nucleic acid purification (BD Biosciences). Total RNA and mRNA were quantified using RiboGreen ${ }^{\circledR}$ RNA quantification (Molecular probes, Eugene, OR, USA). Reverse transcription and PCR were performed in a single tube using the Thermophilus thermus (Tth) reverse transcriptase-DNA polymerase enzyme (GeneAmp ${ }^{\circledR}$ EZ rTth PCR RNA kit; Applied BioSystems, Foster City, CA, USA). However, the protocol was modified for fluorescent detection of amplified products using real-time RT-PCR, whereby the EZ buffer was modified according to a protocol by Roche Applied Science (Indianapolis, IN, USA) to include $50 \mathrm{mM}$ bicine, $115 \mathrm{mM}$ potassium acetate, and $8 \%$ glycerol, pH 8.0 (Sigma, St. Louis, MO, USA). The real-time RT-PCR mixture consisted of $50 \mathrm{ng}$ mRNA dissolved in water, $10 \mu \mathrm{L}$ modified EZ buffer, 5 $\mu \mathrm{L} 25 \mathrm{mM} \mathrm{Mn}(\mathrm{OAc})_{2}, 1$ $\mathrm{U} / \mu \mathrm{L}$ AmpErase ${ }^{\circledR}$ uracil $\mathrm{N}$-glycosylase $\quad\left(\mathrm{SYBR}^{\circledR}\right.$ Green PCR core reagent; Applied Biosystems), $1 \mu \mathrm{L}$ dGACU mixture (SYBR Green PCR core reagent), $2.5 \mathrm{U}$ Tth, $10 \mathrm{ng}$ of each primer, $1 \mu \mathrm{L} 20 \times$ SYBR nucleic acid gel stain (Molecular Probes), and water to a final volume of $50 \mu \mathrm{L}$. Primers for IL-2 and GAPDH have been previously described (19). Reverse transcription was performed at $55^{\circ} \mathrm{C}$ for 40 min for both IL-2 and GAPDH primers, followed by 50 PCR cycles, consisting of denaturation at $94^{\circ}$ for $30 \mathrm{~s}$, annealing at $55^{\circ}$ for $30 \mathrm{~s}$, and extension at $72^{\circ} \mathrm{C}$ for $40 \mathrm{~s}$. The samples were run using a GeneAmp 5700 sequence detector (Applied Biosystems) and analyzed with the software provided. As a positive control, IL-1 $\beta$-specific sequences inserted into the pAW109 RNA were amplified in parallel using the primers provided with the reverse transcription kit. The samples were run in duplicate in at least two independent reactions. The cycle threshold $\left(C_{t}\right)$ was set at 1.2 so that an increase in fluorescence over this value was considered the initiation of log target amplification. Negative PCR controls consisted of amplifying water samples with no target cDNA. Universal precautions were exercised to avoid product contamination.

\section{RESULTS}

\section{Detection of HIV-1-Infected Cells by Anti-gp41 Monoclonal Antibodies}

To determine the capacity of gp41specific MAbs to detect HIV-1-infected cells and to compare the sensitivity of HIV detection using gp41 versus p24 antibodies, H9-IIIB (a chronically infected cell line) or H9 (uninfected parental cell line of H9-IIIB) cells were stained for expression of cell-associated gp41 using $5 \times$ pooled MAbs (2F5, 5F3, F240, 50-69, and 240-D) and for intracellular expression of p24 using commercially available anti-p24 antibody (Beckman Coulter). Nonspecific staining of p24 was at $4 \%$, as determined from $\mathrm{p} 24$-stained uninfected H9 cells (Figure 1A). Approximately 96\% of H9-IIIB cells stained positive for p24 (Figure 1B). On the other hand, the pooled $5 \times$ anti-gp41 MAbs were capable of detecting $68 \%$ of the infected cells (Figure 1D), given that $4 \%$ of uninfected $\mathrm{H} 9$ cells also stained positive for gp41 (Figure 1C). Note that both $\mathrm{p} 24$ and gp41 detected a minority of CD4-negative H9-IIIB cells (Figure 1, B and D), possibly reflecting the reported $\mathrm{CD} 4$ down-modulation by HIV-1 replication (20). Increasing the amount of the pooled anti-gp41 MAbs from $1 \times$ combination $(4.6 \mu \mathrm{g} 2 \mathrm{~F} 5,0.5$ $\mu \mathrm{g}$ F240, $0.5 \mathrm{ng} 240-\mathrm{D}$, and $15 \mu \mathrm{g}$ 5F3, plus a 5-fold dilution of 50-69) to a $6 \times$ combination enhanced gp41 detection of H9-IIIB in a dose-dependent fashion (Figure 1E). Evaluating the ability of each antibody alone versus the pooled anti-gp41 antibodies to detect HIV gp41-positive cells demonstrated that the pooled antibodies were more sensitive in cell surface gp41 detection than any one antibody alone (Figure $1 \mathrm{~F}$ ).

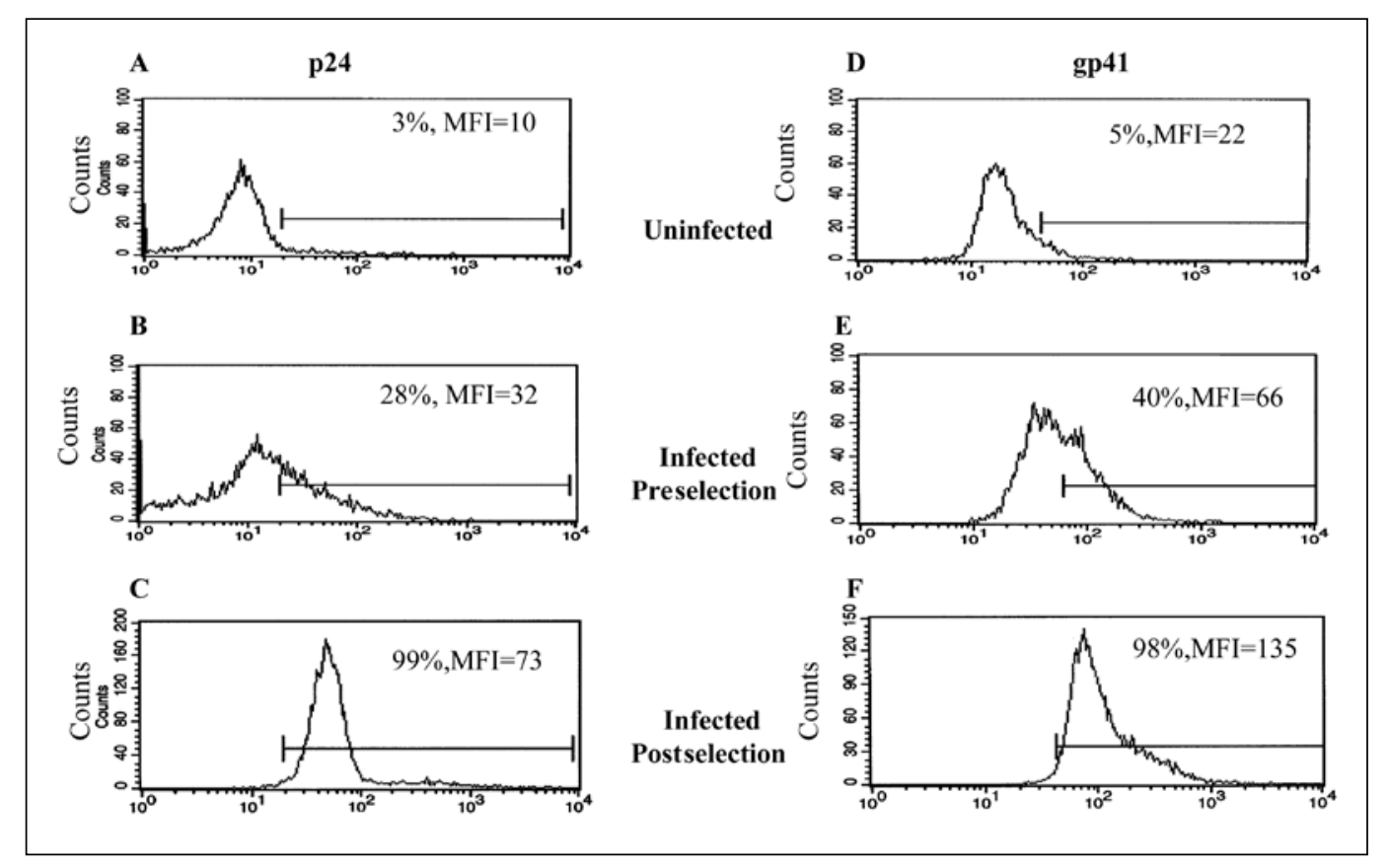

Figure 2. Enrichment of human immunodeficiency virus type 1 (HIV-1)-infected cells by anti-HIV-1 gp41 monoclonal antibodies (MAbs). Uninfected and acutely infected $\mathrm{H} 9$ cells 7 days post-infection were stained with pooled anti-gp41 MAbs, and gp41-positive cells were isolated by indirect magnetic bead selection. All infected fractions (preselection and postselection) were stained for (B and C) HIV p24 and (E-G) gp41. Uninfected cells were used to set the background level for (A) HIV p24 and (D) HIV gp41 staining. MFI, mean fluorescence intensity. 
Antibodies 240-D and 50-69 were the weakest in anti-gp41 detection (data not shown).

\section{Enrichment of HIV-1-Infected T Cell Line by Indirect Anti-gp41 Immunoselection}

To enrich for HIV-positive cells, $8-10 \times 10^{6} \mathrm{H} 9$ cells were infected with HIV IIIB. Eight days post-infection, the cells were labeled with anti-gp41 antibodies and then with anti-human antibodies coupled to microbeads. The anti-gp41 microbead-conjugated cells were isolated by positive selection by running the labeled cells through mag- netic columns, using the autoMACS system. The enrichment efficiency was estimated by immunostaining for gp41 and/or p24 in the different fractions (initial, negative, and positive fractions). Figure 2 shows that uninfected cells had a background of p24 and gp41 staining at $3 \%$ and $5 \%$, respectively (Figure $2, \mathrm{~A}$ and D). Infected cultures before selection were $28 \%$ and $40 \%$ positive for intracellular p24 and cell surface gp41 expression, respectively (Figure 2, B and E). After the gp41 selection, the cells were highly enriched for HIV expression, as determined by $98 \%$ of the cells expressing gp41 and p24 (Figure 2, C and F). The HIV-positive enriched fraction was approximately $80 \%$ viable, as evaluated by trypan blue exclusion assay, and the negative fraction retained approximately $1 \%$ gp41 and p24-positive cells (data not shown).

\section{Enrichment of HIV-1-Infected Peripheral Blood Mononuclear Cells by Indirect Anti-gp41 Immunoselection}

To evaluate the efficacy of this approach for isolating primary cells infected with either a primary isolate or T-tropic isolate of HIV, $10 \times 10^{6}$ PBMCs were infected with primary strain 302151 or IIIB at $20 \mathrm{ng}$ p24/1 $\times$

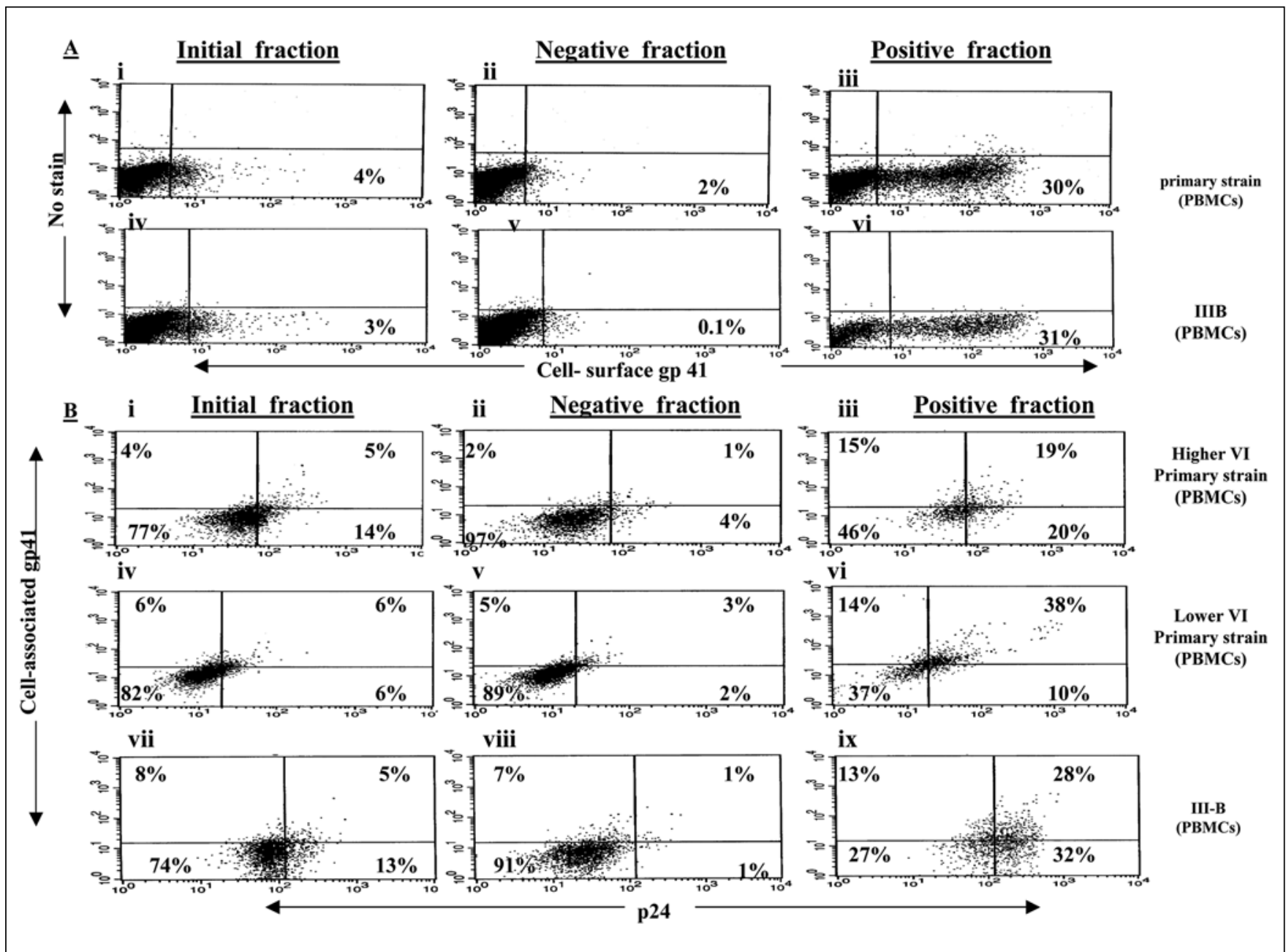

Figure 3. Enrichment of human immunodeficiency virus type 1 (HIV-1)-infected peripheral blood mononuclear cells (PBMCs) using human anti-HIV1 gp41 monoclonal antibodies (MAbs) conjugated to magnetic beads. Total PBMCs were isolated from healthy donors and infected with a primary (HIV strain 302151) or a laboratory-adapted strain (III-B) at $20 \mathrm{ng}$ p24/1 $\times 10^{6}$ cells. (A) Pooled MAbs were conjugated to microbeads and used in positive immunoselection. PBMCs were infected with the primary strain at higher viral inoculum (VI) at $20 \mathrm{ng} \mathrm{p} 24 / 1 \times 10^{6}$ cells or lower VI at $5 \mathrm{ng}$ p $24 / 1 \times 10^{6}$ cells or with HIV IIIB at higher VI. Anti-gp41 immunoselction was performed as described in the text. The fraction used for selection is indicated as the "initial fraction," and the eluted fractions are indicated as the "negative fraction" or "positive fraction." All fractions were stained for (A-i-vi) cell surface gp41 or for (B i-ix) intracellular gp41 and p24. Percentages of positive expression are indicated within each quadrant. 
$10^{6}$ cells. Infected cells were pulled out using pooled anti-gp41 MAbs indirectly conjugated to microbeads and positively selected as described earlier. The initial fraction contained about $4 \%$ or $3 \%$ gp41-positive PBMCs infected with strain 302151 or IIIB, respectively (Fig- ure $3 \mathrm{~A}, \mathrm{i}$ and iv). After positive selection using the anti-gp41 MAb pool, approximately $30 \%$ of the cells were positive for cell surface gp41 (Figure 3A, iii and vi), while the negative fraction contained $2 \%$ or $0.1 \%$ gp41-positive PBMCs, based on whether the cells were infected with strain 302151 or IIIB, respectively (Figure 3A, ii and v).

To evaluate if this enrichment can be impacted by the infecting dose of the virus, PBMCs were infected with 302151 or IIIB at higher $(20 \mathrm{ng}$ p $24 / 1 \times$ $10^{6}$ cells $)$ and lower $\left(5 \mathrm{ng}\right.$ p24/1 $\times 10^{6}$

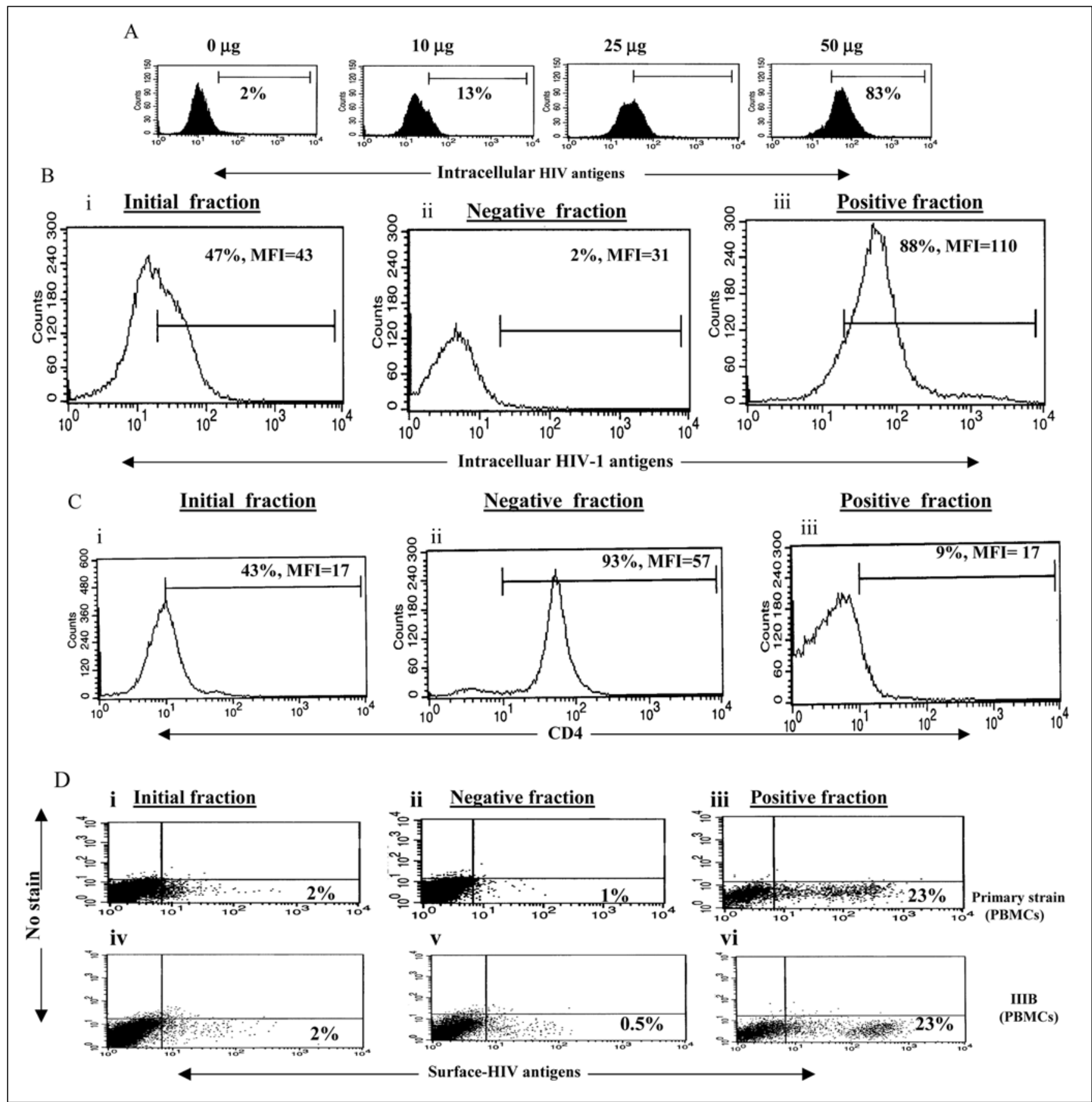

Figure 4. Enrichment of human immunodeficiency virus type 1 (HIV-1)-infected cells by anti-HIV immunoglobulins (HIV-Igs). (A) HIV-Igs from human plasma at 0-50 $\mu \mathrm{g}$ were used to immunostain H9-IIIB cells. (B) HIV-Ig at $50 \mu \mathrm{g}$ was used in the positive selection process, as described previously. (C) Surface expression of CD4 on the three fractions of H9 infected with IIIB was evaluated by flow cytometry. Peripheral blood mononuclear cells (PBMCs) were infected with the primary strain, H9 cells were infected with IIIB, and $50 \mu \mathrm{g}$ HIV-Ig were used in the positive selection process. (D) Percentages of expression of HIV-positive cells were evaluated by immunostaining using human Ig. MFI, mean fluorescence intensity 
cells) viral inoculum and co-stained for gp41 and p24. Dual staining increases the sensitivity detecting HIV-positive population. The level of HIV-positive enrichment was 1.0-fold higher in cells infected with low viral inoculum than higher viral inoculum. Specifically, comparing all HIV-positive fractions (i.e., gp41 or p24 or both gp41 and p24) indicated the selection from the higher viral inoculum cells resulted in 54\% HIV-positive cells (represents all three HIV-positive quadrants), while the selection of lower viral inoculum cells resulted in 62\% HIV-positive cells (Figure $3 \mathrm{~B}$, iii and vi). HIV-positive cells remaining in the negative/unselected fraction ranged from $7 \%-10 \%$, depending on the viral inoculum and HIV strain used (Figure 3B, ii, v, and viii). We also enriched for PBMCs infected with HIV IIIB by $73 \%$, while $9 \%$ of the infected cells remained in the negative fraction (Figure 3B, viii and ix).

Because the described anti-gp41 MAbs may be difficult to acquire by some investigators, we evaluated the capacity of HIV-Ig, representing polyclonal anti-HIV antibodies prepared from human plasma, to be used in this selection process. First, we evaluated the ability of HIV-Ig to detect H9-IIIB cells using varying amounts of HIV-Ig. Increasing the amount of HIV-Ig from 10-50 $\mu \mathrm{g}$ enhanced the sensitivity of HIV antigen detection in a dose-dependent manner, with $50 \mu \mathrm{g}$ HIV-Ig detecting $83 \%$ of infected cells (Figure 4A). Using $50 \mu \mathrm{g}$ HIV-Ig to positively select for $\mathrm{H} 9$ cells infected with IIIB resulted in the enrichment of infected cells by $88 \%$ from an initial $47 \%$ infected population, while the negative fraction retained only $2 \%$ of the infected cells (Figure 4B). Examining CD4 expression on infected H9 cells from the various fractions (initial, negative, or positive) indicated a substantial down-regulation of CD4 expression in the positively infected cells, as would have been expected. (Figure 4C). Using this polyclonal antibody strategy was moderately effective in enriching for HIV-infected PBMCs, at similar levels, whether the cells were infected with 302151 or IIIB (Figure 4D), although the efficiency was less than that observed with IIIB-infected H9 cells.

\section{Interleukin 2 mRNA Level Is Higher in gp41-Positive Cells}

To establish that the fractionated cells can be used in other assays and to specifically evaluate if HIV-positive cells have modulated IL-2 expression, mRNA was isolated from gp41-positive and uninfected H9 cells, and the IL-2 transcript level was evaluated by real-time RT-PCR. IL-2 mRNA expres- sion from gp41-positive cells was amplified at much earlier cycle numbers (mean $\mathrm{C}_{t}=24$ ) than uninfected cells (mean $\mathrm{C}_{\mathrm{t}}=30$ ), as indicated by three experiments (Figure 5A; $P=0.005$ ), while the housekeeping gene GAPDH consistently amplified at mean $\mathrm{C}_{t}=17$ from both the gp41-positive selected fraction and uninfected fraction. Intracellular staining for IL-2 from the three fractions demonstrated enhanced IL-2 
expression in the $\mathrm{p} 24$-positive fraction by $24 \%$ in comparison to $3 \%$ in the initial faction and $1 \%$ in the negative fraction (Figure 5B).

\section{DISCUSSION}

Devising effective technical approaches to isolate or enrich HIV-infected cells allows for further studies to evaluate the impact of HIV infection on cells at the molecular level. Such an approach needs to discriminate between infected and uninfected cells and, ideally, should be relatively inexpensive, time-efficient, and lead to the isolation of viable HIV-positive cells. Because $\mathrm{HIV}$-infected cells retain the expression of HIV gp41 on their surface after infection, we used a conventional assay based on anti-gp41 MAbs or HIV-Ig indirectly conjugated to magnetic beads to pull out these infected cells. Both
HIV-Ig and pooled anti-gp41 MAbs selected $>80 \%$ viable HIV-infected cells but at varying efficiencies. The pooled anti-gp41 MAbs were effectively able to enrich for H9 cells infected with a laboratory-adapted isolate of HIV (IIIB). The level of enrichment was up to $98 \%$ with $>1 \%$ of H9-IIIB-infected cells remaining in the negative fraction. The enrichment of PBMCs infected with either a primary or IIIB isolate was less efficient, at $54 \%$, when the initial viral inoculum was high. However, at lower viral inoculums, the enriching efficiency was better for primary-infected PBMCs than at higher viral inoculum. The selection process was also more efficient for IIIB-infected PBMCs than primary-infected PBMCs, regardless of the inoculum dose used. The variation in selection purity is unlikely due to variation in gp41 cell surface expression on primary cells infected with primary isolates because the percentage of expression of gp41 was similar between H9 and PBMCs. However, it could be due to the overall lower frequency of HIV infection of primary cells in comparison to a $\mathrm{T}$ cell line.

Using HIV-Igs, (anti-HIV serum) in the positive selection process was also highly efficient for selecting HIV H9-IIIB-infected cells. However, again the frequency of enriching for primary cells (PBMCs) infected with either a primary strain or a laboratory-adapted strain was less efficient than an infected $\mathrm{T}$ cell line. In general, the pooled anti-gp41 MAbs were more sensitive for recognizing $\mathrm{HIV}$-infected cells than HIV-Ig. This observation may be because the pooled anti-gp41 MAbs recognize different epitopes of gp41, which possibly leads to enhanced antigen recognition.

By obtaining two fractions (depleted and enriched in HIV-1-infected cells) from the initial HIV infected culture, our system provides the opportunity to discriminate between direct viral effect requiring viral presence or indirect effect occurring in cells exposed to viral products or expressing the virus at lower rate. For instance, the HIVIg-based magnetic immunoselection protocol revealed considerable enrichment of CD4 cells in the positive selection, which clearly confirms that CD4 loss was due to active viral replication.

Having isolated an enriched population of HIV-positive cells, we examined the profile of IL-2 mRNA of HIVpositive cells using realtime RT-PCR. Enriched HIV-positive cells expressed elevated levels of IL-2 mRNA. We have also observed higher HIV expression within IL-2-positive cells using intracellular staining for IL-2. Previous reports have documented that HIV increases IL-2
Figure 5. Comparison of interleukin 2 (IL-2) mRNA levels and protein expression between the gp41-positive fraction and the initial fraction. mRNA was isolated from both the uninfected and the gp41-positive fraction, and real-time reverse transcription PCR (RT-PCR) was performed for both IL-2 and GAPDH mRNA. All samples were assayed in duplicate in three independent experiments. (A) The mean amplification cycle of the gp41-positive selection fraction and the uninfected cells for IL-2 and GAPDH transcript. The difference in IL-2 mRNA between uninfected and infected cells is significant $(P$ $=0.005$ ), as determined by the paired Student's $t$ test. (B) Flow cytometric histogram of the percentage of IL-2 intracellular protein expression in the initial, negative, and gp41-positive fractions. 
expression at the transcriptional level (21-23). It is unclear, however, if HIV specifically targets IL-2 expressing cells, or if HIV up-regulates IL-2 postexpression, or perhaps both IL-2 high and low expressing cell populations are equally infected but that a higher activation state, as measured by IL-2 expression, could result in greater expression of viral gene products. Examination of IL-2 enhanced expression in HIV-positive population indicated that IL-2 induction is correlated with higher cell activation and higher expression of the anti-apoptotic Bcl-2 protein among infected cells (B. Bahbouhi and L. AlHarthi, manuscript submitted), which seems to support the latter rationale. Although the examination of IL-2 expression from HIV-enriched fractions using real-time RT-PCR did not require that the cells remain viable, this approach does generate viable cells that can be further cultured and analyzed.

While the effect of viral (e.g., Tat, $\mathrm{Vpr}$, and Nef) proteins can be easily studied by their addition to uninfected cells as previously described (24-26), analyzing the direct effect of the virus on cells requires the ability to pull out enriched infected cells. The approach we describe here, which uses anti-gp41 MAbs or HIV-Ig indirectly conjugated to microbeads to generate an enriched infected population, is ideal for tracking the effect of the virus on given host cell gene expression. It is also ideal for $\mathrm{T}$ cells infected with HIV, but a higher proportion of infected PBMCs may also be enriched. This method may be optimized for selecting HIVinfected cells by generating anti-gp41 MAbs that are directly conjugated to fluorochrome and microbeads. Such a modification would probably lead to a higher yield of HIV-infected cells and to shorten the length of the protocol.

\section{REFERENCES}

1.Cloyd, M.W., J.J. Chen, P. Adeqboyega, and L. Wang. 2001. How does HIV cause depletion of CD4 lymphocytes? A mechanism involving virus signaling through its cellular receptors. Curr. Mol. Med. 1:545-550.

2.Cloyd, M.W., J.J. Chen, and I. Wang. 2000. How does HIV cause AIDS? The homing theory. Mol. Med. Today 6:108-111.

3.Uittenbogaart, C.H., D.J. Anisman, J.A. Zack, A. Economides, I. Schmid, and E.F.
Hays. 1994. Effects of cytokines on HIV-1 production by thymocytes. Thymus $23: 155$ 175.

4.O'Brien, W.A., K. Grovit-Ferbas, A. Namazi, S. Ovcak-Derzic, H.J. Wang, J. Park, C. Yeramian, S.H. Mao, et al. 1995. Human immunodeficiency virus-type 1 replication can be increased in peripheral blood of seropositive patients after influenza vaccination. Blood 86:1082-1089.

5.Liu, X., J. Zha, J. Nishitani, H. Chen, and J.A. Zack. 2003. HIV-1 infection in peripheral blood lymphocytes (PBLs) exposed to alcohol. Virology 307:37-44.

6.Savarino, A., L. Gennero, K. Sperber, and J.R. Boelaert. 2001. The anti-HIV-1 activity of chloroquine. J. Clin. Virol. 20:131-135.

7.Yoo, J., H. Chen, T. Kraus, D. Hirsch, S. Polyak, I. George, and K. Sperber. 1996. Altered cytokine production and accessory cell function after HIV-1 infection. J. Immunol. 157:1313-1320.

8.Boelaert, J.R., K. Sperber, and J. Piette. 2001. The additive in vitro anti-HIV-1 effect of chloroquine, when combined with zidovudine and hydroxyurea. Biochem. Pharmacol. 61:1531-1535.

9.Grivel, J.C., A. Biancotto, Y. Ito, R.G. Lima, and L.B. Margolis. 2003. Bystander CD4+ T lymphocytes survive in HIV-infected human lymphoid tissue. AIDS Res. Hum. Retroviruses 19:211-216.

10.Azad, A.A. 2000. Could Nef and Vpr proteins contribute to disease progression by promoting depletion of bystander cells and prolonged survival of HIV-infected cells? Biochem. Biophys. Res. Commun. 267:677-685.

11.Jordan, A., D. Bisgrove, and E. Verdin. 2003. HIV reproducibly establishes a latent infection after acute infection of $\mathrm{T}$ cells in vitro. EMBO J. 22:1868-1877.

12.Muthumani, K., L.J. Montaner, V. Ayyavoo, and D.B. Weiner. 2000. Vpr-GFP virion particle identifies HIV-infected targets and preserves HIV-1Vpr function in macrophages and T-cells. DNA Cell Biol. 19:179-188.

13.Bolton, D.L., B.I. Hahn, E.A. Park, L.L. Lehnhoff, F. Hornung, and M.J. Lenardo. 2002. Death of CD4(+) T-cell lines caused by human immunodeficiency virus type 1 does not depend on caspases or apoptosis. J. Virol. 76:5094-5107.

14.Jamieson, B.D., S. Pang, G.M. Aldrovandi, J. Zha, and J.A. Zack. 1995. In vivo pathogenic properties of two clonal human immunodeficiency virus type 1 isolates. J. Virol. 69: 6259-6264.

15.Mascola, J.R., M.K. Louder, C. Winter, R. Prabhakara, S.C. De Rosa, D.C. Douek, B.J. Hill, D. Gabuzda, et al. 2002. Human immunodeficiency virus type 1 neutralization measured by flow cytometric quantitation of single-round infection of primary human $\mathrm{T}$ cells. J. Virol. 76:4810-4821.

16.Doms, R.W. and J.P. Moore. 2000. HIV-1 membrane fusion: targets of opportunity. J. Cell Biol. 151:F9-F14.

17.Land, A. and I. Braakman. 2001. Folding of the human immunodeficiency virus type 1 envelope glycoprotein in the endoplasmic reticulum. Biochimie 83:783-790.

18.Boyd, J.E. and K.B. James. 1992. Cell responses to HIV and the development of human monoclonal antibodies. Clin. Exp. Immunol. 88:189-202.

19.Kotake, S., H.R. Schumacher, Jr., and R.L. Wilder. 1996. A simple nested RT-PCR method for quantitation of the relative amounts of multiple cytokine mRNAs in small tissue samples. J. Immunol. Methods 199:193-203.

20.Tanaka, M., T. Ueno, T. Nakahara, K. Sasaki, A. Ishimoto, and H. Sakai. 2003. Downregulation of CD4 is required for maintenance of viral infectivity of HIV-1. Virology 311:316-325.

21.Westendorp, M.O., M. Li-Weber, R.W. Frank, and P.H. Krammer. 1994. Human immunodeficiency virus type 1 Tat upregulates interleukin-2 secretion in activated $\mathrm{T}$ cells. J. Virol. 68:4177-4185.

22.Ott, M., S. Emiliani, C. Van Lint, G. Herbein, J. Lovett, N. Chirmule, T. McCloskey, S. Pahwa, et al. 1997. Immune hyperactivation of HIV-1-infected T cells mediated by Tat and the CD28 pathway. Science 275: 1481-1485.

23.Vacca, A., M. Farina, M. Maroder, E. Alesse, I. Screpanti, L. Frati, and A. Gulino. 1994. Human immunodeficiency virus type-1 tat enhances interleukin-2 promoter activity through synergism with phorbol ester and calcium-mediated activation of the NFAT cis-regulatory motif. Biochem. Biophys. Res. Commun. 205:467-474.

24.Eckstein, D.A., M.P. Sherman, M.L. Penn, P.S. Chin, C.M. De Noronha, W.C. Greene, and M.A. Goldsmith. 2001. HIV-1 Vpr enhances viral burden by facilitating infection of tissue macrophages but not nondividing CD4+ T cells. J. Exp. Med. 194:1407-1419.

25.Ott, M., J.L. Lovett, L. Mueller, and E. Verdin. 1998. Superinduction of IL-8 in T cells by HIV-1 Tat protein is mediated through NFkappaB factors. J. Immunol. 160:2872-2880.

26.Jenkins, Y., M. McEntee, K. Weis, and W.C. Greene. 1998. Characterization of HIV-1 vpr nuclear import: analysis of signals and pathways. J. Cell Biol. 143:875-885.

Received 30 July 2003; accepted 22 October 2003.

\section{Address correspondence to:}

Lena Al-Harthi

Rush University Medical Center

Department of Immunology/Microbiology 1653 West Congress Parkway, Rm. 1577 JSC

Chicago, IL 60612, USA

e-mail: lalharth@rush.edu 